\title{
CORRIGENDUM
}

\section{NMR analyses of compatible solutes in a halotolerant Brevibacterium sp.}

Shinichi Nagata, Kyoko Adachi and Hiroshi Sano

Microbiology (1996), 142, 3355-3362

In Table 2, p. 3360, the values given for 'Amino acids' and 'Total' in the top and bottom rows were incorrect, and the words 'and hydroxyproline' were missing from the footnote. The correct version of the table is given below.

Table 2. Internal solute and ion concentrations (mM) in Brevibacterium sp. grown in $\mathrm{C}$ medium with different $\mathrm{NaCl}$ concentrations

\begin{tabular}{|lcccccc|}
\hline $\mathrm{NaCl}$ concn $(\mathbf{M}) \ldots$ & \multicolumn{1}{c}{$\mathbf{0}$} & $\mathbf{0} \cdot \mathbf{1}$ & $\mathbf{0 \cdot 5}$ & $\mathbf{1 \cdot 0}$ & $\mathbf{1 \cdot 5}$ \\
\hline Amino acids* & $89 \cdot 4$ & $97 \cdot 2$ & $124 \cdot 6$ & $140 \cdot 0$ & $198 \cdot 2$ & $149 \cdot 7$ \\
Glutamate & $134 \cdot 7 \pm 13 \cdot 6$ & $193 \cdot 7 \pm 3 \cdot 3$ & $241 \cdot 4 \pm 29 \cdot 3$ & $209 \cdot 5 \pm 13 \cdot 3$ & $193 \cdot 3 \pm 9 \cdot 8$ & $156 \cdot 9 \pm 0 \cdot 5$ \\
Glycine betaine & $99 \cdot 2 \pm 5 \cdot 1$ & $94 \cdot 9 \pm 10 \cdot 4$ & $136 \cdot 3 \pm 28 \cdot 1$ & $126 \cdot 0 \pm 24 \cdot 1$ & $135 \cdot 0 \pm 1 \cdot 2$ & $140 \cdot 5 \pm 4 \cdot 8$ \\
Ectoine & $0 \cdot 0$ & $0 \cdot 0$ & $108 \cdot 5 \pm 1 \cdot 3$ & $252 \cdot 1 \pm 18 \cdot 1$ & $385 \cdot 3 \pm 8 \cdot 9$ & $480 \cdot 3 \pm 5 \cdot 2$ \\
Hydroxyectoine & $0 \cdot 0$ & $0 \cdot 0$ & $0 \cdot 0$ & $0 \cdot 0$ & $4 \cdot 8 \pm 0 \cdot 1$ & $63 \cdot 9 \pm 3 \cdot 7$ \\
Hydroxyproline & $44 \cdot 5 \pm 3 \cdot 9$ & $51 \cdot 4 \pm 5 \cdot 8$ & $59 \cdot 6 \pm 15 \cdot 3$ & $97 \cdot 9 \pm 17 \cdot 6$ & $155 \cdot 5 \pm 0 \cdot 9$ & $65 \cdot 6 \pm 11 \cdot 7$ \\
TMAH & $66 \cdot 7 \pm 2 \cdot 8$ & $94 \cdot 7 \pm 4 \cdot 5$ & $123 \cdot 6 \pm 5 \cdot 1$ & $110 \cdot 9 \pm 12 \cdot 9$ & $111 \cdot 5 \pm 10 \cdot 1$ & $116 \cdot 8 \pm 10 \cdot 6$ \\
Na & $0 \cdot 0$ & $16 \cdot 2 \pm 7 \cdot 2$ & $35 \cdot 5 \pm 4 \cdot 7$ & $43 \cdot 4 \pm 7 \cdot 7$ & $77 \cdot 2 \pm 1 \cdot 1$ & $117 \cdot 0 \pm 18 \cdot 8$ \\
$\mathrm{~K}^{+}$ & $288 \cdot 1$ & $297 \cdot 8$ & $364 \cdot 4$ & $372 \cdot 9$ & $350 \cdot 1$ & $327 \cdot 9$ \\
Total & $722 \cdot 6$ & $845 \cdot 9$ & $1193 \cdot 9$ & $1352 \cdot 7$ & $1610 \cdot 9$ & $1618 \cdot 6$ \\
\hline
\end{tabular}

* Total amounts of amino acids, excluding glutamate and hydroxyproline, determined by amino acid analysis (Mimura et al., 1994). 